\title{
Ovine prion protein genotype frequencies in northwestern China
}

\author{
C.-L. Zhao ${ }^{1}$, R. Wu ${ }^{1,2}$, L. Liu ${ }^{1}$, F.-D. Li ${ }^{3}$, X.-L. Zhang ${ }^{1}$, C. Wang ${ }^{1}$, F. Wang ${ }^{1}$, \\ X.-L. Diao' ${ }^{1}$, H.-W. Guan ${ }^{1}$, X. Wang' ${ }^{1}$ and L. Zhou ${ }^{1}$ \\ ${ }^{1}$ College of Veterinary Medicine, Gansu Agricultural University, Lanzhou, China \\ ${ }^{2}$ State Key Laboratory of Veterinary Etiological Biology, \\ Lanzhou Veterinary Research Institute, \\ Chinese Academy of Agricultural Sciences, Lanzhou, China \\ ${ }^{3}$ College of Animal Science and Technology, \\ Gansu Agricultural University, Lanzhou, China \\ Corresponding author: R. Wu \\ E-mail: wurun@gsau.edu.cn
}

Genet. Mol. Res. 11 (2): 1671-1681 (2012)

Received January 30, 2012

Accepted March 14, 2012

Published June 21, 2012

DOI http://dx.doi.org/10.4238/2012.June.21.2

\begin{abstract}
Anti-scrapie breeding programs have been initiated to screen for scrapie-resistant sheep based on ovine prion protein gene (PRNP) genotypes at codons 136, 154 and 171 in many countries, especially European Union member states. However, investigation of sheep PRNP genotypes is limited in China, despite the large number of sheep breeds. We analyzed 432 sheep of five different breeds from farms in northwestern China, using PCR-single-strand conformational polymorphism analysis (PCR-SSCP); the corresponding haplotypes of different PRNP alleles were cloned. PRNP allele genotyping was done by amplification refractory mutation system-PCR (ARMS-PCR), according to the haplotype clones of each PRNP allele. The validity of ARMS-PCR was checked by PCR-SSCP. Another 325 unknown PRNP genotypes of other sheep breeds were analyzed according to the established ARMSPCR. Genotype frequencies of 757 sheep were analyzed with these two methods to evaluate susceptibility to scrapie in northwestern China.
\end{abstract}


Relevant mutations were also detected at other sites. Both methods were effective for ovine PRNP allele genotyping, and the results of the analysis completely coincided. Scrapie-resistant genotypes were found to be uncommon, indicating a high risk for ovine scrapie in northwest China. In addition to codons 136, 154 and 171, we found numerous new mutations; nearly half of them were previously unreported. These sheep populations have a high degree of polymorphism at the PRNP locus.

Key words: Sheep; PRNP alleles; Genotyping; PCR-SSCP; ARMS-PCR; Mutations

\section{INTRODUCTION}

Scrapie, a member of the mammalian transmissible spongiform encephalopathy disease family, is transmissible between animals via an isoform of the prion protein. Previous studies confirmed that sheep scrapie was associated with PRNP genotypes, and controlled by sheep PRNP polymorphisms at codons 136, 154 and 171 (Hunter, 1997; Andreoletti et al., 2006; Goldmann et al., 2006). Accordingly, selecting scrapie-resistant sheep by detecting scrapie-resistant genotype frequencies may gradually decrease the risk of scrapie. Therefore, anti-scrapie breeding programs have been established in European Union member states, and the occurrence of scrapie has been controlled (Hagenaars et al., 2006; Melchior et al., 2010). Given that there was no significant relationship between ovine PRNP genotypes and sheep product traits (De Vries et al., 2004; Sawalha et al., 2007; Sweeney et al., 2007; Vitezica et al., 2007; Lipsky et al., 2008), the anti-scrapie breeding program is of great significance in the prevention of scrapie.

However, in China, there are no anti-scrapie breeding programs and the main varieties of sheep PRNP genotype frequency are unclear now. Although there has not been any scrapie cases reported in China, previous studies showed that there is high risk of scrapie in the sheep population of China (Li and Tian, 2002; Zhang et al., 2004). Therefore, it is very important to investigate the scrapie PRNP for scrapie prevention and sheep anti-scrapie breeding in China.

In the present study, we analyzed the distribution of PRNP genotypes and mutations at other codons in a total of 727 sheep using the PCR-SSCP and ARMS-PCR methods, and evaluated scrapie risk in northwestern China.

\section{MATERIAL AND METHODS}

\section{Animals and samples}

The major sheep breeds (total of 757 sheep) in northwestern China were studied: Small Tail Han sheep $(\mathrm{N}=104)$, Qiaoke sheep $(\mathrm{N}=88)$, Oula sheep $(\mathrm{N}=37)$, Mongolian sheep $(\mathrm{N}=$ 99), Tan sheep $(\mathrm{N}=100)$, Gansu Alpine Merino $(\mathrm{N}=139)$, and Gansu modern meat sheep new population $(\mathrm{N}=190)$.

Blood samples were treated with acid citrate dextrose, and genomic DNA was extracted using the Blood Genome DNA Extraction kit (TaKaRa). 


\section{Determination of the PRNP gene with PCR-SSCP}

\section{Primer design and PCR amplification}

SSCP primers were designed based on the sequence (AY723289) submitted by our laboratory to amplify a 268-bp fragment of the ovine PRNP gene, which covered three important codons for scrapie susceptibility. Upstream primer corresponding to codons 121 to 126 was UP (5'-AGCTGGAGCAGTGGTAGGG-3'); downstream primer corresponding to codons 203 to 209 was DN (5'-CATTATCTTGATGTCAGTTTCG-3'). PCR amplifications were performed in a $25-\mu \mathrm{L}$ reaction volume containing $1.0 \mu \mathrm{L}$ genomic DNA, $10 \mathrm{pM}$ each primer, and $12.5 \mu \mathrm{L}$ Premix $\operatorname{Ex~Taq~}^{\circledR}(\mathrm{TaKaRa})$. The amplification reactions were placed in a MyCycler ${ }^{\mathrm{TM}}$ thermal cycler (BIO-RAD Laboratories) and consisted of denaturation at $94^{\circ} \mathrm{C}$ for $5 \mathrm{~min}$, followed by 35 cycles of $94^{\circ} \mathrm{C}$ for $45 \mathrm{~s}, 60^{\circ} \mathrm{C}$ for $45 \mathrm{~s}$ and $72^{\circ} \mathrm{C}$ for $45 \mathrm{~s}$. This was followed by a final extension step at $72^{\circ} \mathrm{C}$ for $5 \mathrm{~min}$. The products were visualized on a $15 \mathrm{~g} / \mathrm{L}$ agarose gel. The samples analyzed with PCR-SSCP were: 104 of Small Tail Han sheep, 88 of Qiaoke sheep, 99 of Mongolian sheep, 100 of Tan sheep, and 41 of Gansu alpine merino, for a total of 432 blood samples.

\section{SSCP analysis of genome amplification products}

A volume of $2.0 \mu \mathrm{L}$ of each amplified product was mixed with $8.0 \mu \mathrm{L}$ loading dye ( $98 \%$ deionized formamide, $10 \mathrm{mM}$ EDTA, $0.025 \%$ bromophenol blue, $0.025 \%$ xylene-cyanol), and after denaturation at $95^{\circ} \mathrm{C}$ for $10 \mathrm{~min}$, samples were rapidly cooled on wet ice and then loaded on $12 \%$ acrylamide:bisacrylamide (49:1; Amresco) gels without glycerol. Electrophoresis was performed at $200 \mathrm{~V}$ for $45 \mathrm{~h}$ at $4^{\circ} \mathrm{C}$ in $0.5 \mathrm{X}$ TBE buffer, and gels were silver-stained (Sanguinetti et al., 1994).

\section{Screening of PRNP allele clones}

The PCR amplification products were cloned into pGEM-T Vector (Promega), and transformed into competent Escherichia coli cells. Three clones of each sample with two bands of SSCP analysis were selected, while ten clones were selected for the samples with three or four bands. These clones were incubated overnight at $37^{\circ} \mathrm{C}$ in a shaking rotary incubator, and plasmids were recovered from bacterial cells using Plasmid Mini kits (BioDev-Tech. Co., Ltd.).

The amplified products from plasmid DNA were analyzed with corresponding products from genomic DNA under the same conditions using SSCP. Two of the different PRNP haplotype clones of each allele were screened with genomic PCR products of the SSCP banding patterns as references. One clone of each PRNP allele homozygous sheep and two clones of each heterozygote sheep were sequenced by biological company (Invitrogen), and sequence analysis was performed with BioEdit. PRNP genotypes were then determined according to the sequencing results, and PRNP genotype distribution and the mutations in other codons, namely 136, 154 and 171, were analyzed.

\section{ARMS genotyping}

\section{Primer design and PCR optimization}

ARMS primers of all the mutations at codons 136, 154 and 171 were designed using 
different plasmids of PRNP haplotype clones on the basis of ARMS principle. Primers of mutations at codon 136 were named $136 \mathrm{Ap}, 136 \mathrm{Vp}$ and $136 \mathrm{Tp}$, and primers of mutations at 154 and 171 were named $154 \mathrm{Rp}, 154 \mathrm{Hp}, 154 \mathrm{Lp}$ and $171 \mathrm{Qp}, 171 \mathrm{Rp}, 171 \mathrm{Kp}$, and $171 \mathrm{Hp}$, respectively. These ARMS primer sequences, amplicon sizes, and optimized conditions are listed in Table 1. The annealing temperature of each primer was also optimized. Amplified products (about 7.35 $\mathrm{mM}$ ) with SSCP primers were serially diluted 2-fold from 1/100 to 1/1200, and the best template and ARMS primer concentration was finally used for the following experiments (ARMSPCR conditions for PRNP genotyping are shown in Table 2).

Table 1. ARMS primers of all mutations at codons 136, 154 and 171 designed using different plasmids of PRNP haplotype clones.

\begin{tabular}{llcllr}
\hline ARMS primers & Alleles & Codons & Upstream primer sequences & Downstream primer sequences & Expected size (bp) \\
\hline $136 \mathrm{Ap}$ & A-136 & 136 & GCATGTGGCAGGAGCTGCTG & TGTAGGCCTGCTCATAGC & 63 \\
$136 \mathrm{Vp}$ & V-136 & 136 & GCATGTGGCAGGAGCTGCTG & TAGCTAGGCCTGCTCAAGA & 64 \\
$136 \mathrm{Tp}$ & T-136 & 136 & GCATGTGGCAGGAGCTGCTG & GCTAGGCCTGCTCATTGT & 63 \\
$154 \mathrm{Rp}$ & $\mathrm{R}-154$ & 154 & GCATGTGGCAGGAGCTGCTG & GCTCAACGGTACATGTTTCAC & 122 \\
$154 \mathrm{Hp}$ & $\mathrm{H}-154$ & 154 & GCATGTGGCAGGAGCTGCTG & CGCTAACGGTACATGTTTGAT & 122 \\
$154 \mathrm{Lp}$ & $\mathrm{L}-154$ & 154 & GCATGTGGCAGGAGCTGCTG & CCTCAACGGTACATGTTTCAA & 122 \\
$171 \mathrm{Qp}$ & $\mathrm{Q}-171$ & 171 & GCATGTGGCAGGAGCTGCTG & TGCGACGTCTGGTTACTATCCTG & 174 \\
$171 \mathrm{Rp}$ & $\mathrm{R}-171$ & 171 & GCATGTGGCAGGAGCTGCTG & CTGCTACGTCTGGTTACTATGCC & 173 \\
$171 \mathrm{Kp}$ & K-171 & 171 & GCATGTGGCAGGAGCTGCTG & TGTTCGACGTCTGGTTACTATATT & 174 \\
$171 \mathrm{Hp}$ & H-171 & 171 & GCATGTGGCAGGAGCTGCTG & CTGCGACGTCTGGTTACTGCAA & 175 \\
\hline
\end{tabular}

Primers of mutations at codon 136 were named 136Ap, 136Vp and 136Tp, and primers of mutations at 154 and 171 were named $154 \mathrm{Rp}, 154 \mathrm{Hp}, 154 \mathrm{Lp}$ and $171 \mathrm{Qp}, 171 \mathrm{Rp}, 171 \mathrm{Kp}$, and $171 \mathrm{Hp}$, respectively.

\section{ARMS analysis}

Unknown PRNP genotype sheep, including three breeds (190 of Gansu modern meat sheep new population, 98 of Gansu Alpine Merino, 37 of Oula sheep, amounted to 325) were genotyped by ARMS-PCR. The products amplified from genomic DNA with SSCP primers were diluted, and then, PCR with ARMS primers was performed as indicated in Table 2.

\begin{tabular}{|c|c|c|c|c|c|c|c|c|}
\hline ARMS primers & $\begin{array}{l}\text { Dilution } \\
\text { ratio }\end{array}$ & $\begin{array}{c}\text { Extension } \\
\text { time }(\mathrm{s})\end{array}$ & $\begin{array}{c}\mathrm{Tm} \\
\left({ }^{\circ} \mathrm{C}\right)\end{array}$ & $\begin{array}{c}\text { Cycle } \\
\text { number }\end{array}$ & $\begin{array}{c}\text { Premix Ex } \\
\text { Taq }(\mu \mathrm{L})\end{array}$ & $\begin{array}{l}\text { Diluted PCR } \\
\text { product }(\mu \mathrm{L})\end{array}$ & $\begin{array}{c}\text { Primer of each } \\
10 \mathrm{pM}(\mu \mathrm{L})\end{array}$ & $\begin{array}{c}\mathrm{ddH}_{2} \mathrm{O} \\
(\mu \mathrm{L})\end{array}$ \\
\hline $136 \mathrm{Ap}$ & $1 / 600$ & 30 & 63 & 35 & 12.5 & 1.0 & 1.3 & 8.9 \\
\hline $136 \mathrm{Vp}$ & $1 / 600$ & 30 & 63 & 35 & 12.5 & 1.0 & 1.3 & 8.9 \\
\hline $136 \mathrm{Tp}$ & $1 / 600$ & 30 & 63 & 35 & 12.5 & 1.0 & 1.3 & 8.9 \\
\hline $154 \mathrm{Rp}$ & $1 / 800$ & 30 & 65 & 35 & 12.5 & 1.0 & 1.3 & 8.9 \\
\hline $154 \mathrm{Hp}$ & $1 / 800$ & 30 & 65 & 35 & 12.5 & 1.0 & 1.3 & 8.9 \\
\hline $154 \mathrm{Lp}$ & $1 / 800$ & 30 & 65 & 35 & 12.5 & 1.0 & 1.3 & 8.9 \\
\hline $171 \mathrm{Qp}$ & $1 / 1000$ & 30 & 65 & 35 & 12.5 & 1.0 & 1.3 & 8.9 \\
\hline $171 \mathrm{Rp}$ & $1 / 1000$ & 30 & 65 & 35 & 12.5 & 1.0 & 1.3 & 8.9 \\
\hline $171 \mathrm{Kp}$ & $1 / 1000$ & 30 & 65 & 35 & 12.5 & 1.0 & 1.3 & 8.9 \\
\hline $171 \mathrm{Hp}$ & $1 / 1000$ & 30 & 65 & 35 & 12.5 & 1.0 & 1.3 & 8.9 \\
\hline
\end{tabular}

The annealing temperature of each primer was optimized. Amplified products with SSCP primers were diluted from $1 / 100,1 / 200, \ldots \ldots$, to $1 / 1200$, and the best template and ARMS primers concentration was finally used for next experiments.

\section{Polymorphism analysis and scrapie risk evaluation}

All genotyped sheep, including 432 sheep determined with PCR-SSCP and 325 sheep de- 
termined with ARMS-PCR, were analyzed and the PRNP genotype distribution was investigated. After the genotyping of 757 sheep, the risk of scrapie in northwestern China was evaluated.

\section{RESULTS}

\section{PCR-SSCP analysis}

DNA fragments of PRNP alleles from 432 sheep were amplified and two clones of the haplotypes for each allele were also obtained (Figure 1). After sequenced, PRNP genotypes were determined by PCR-SSCP (Table 3). The major genotypes from all groups were ARQ/ARQ, ARR/ARQ, ARR/ARR, ARQ/ARH, ARH/ARH, ARK/ARK, ARK/ARQ, VRQ/ARQ, TRQ/ARQ, ARQ/AHQ, ARK/ARR, ARK/ARH, TRQ/ARR, AHR/ARQ, ARR/ARH, TRQ/TRQ, AHQ/AHQ.

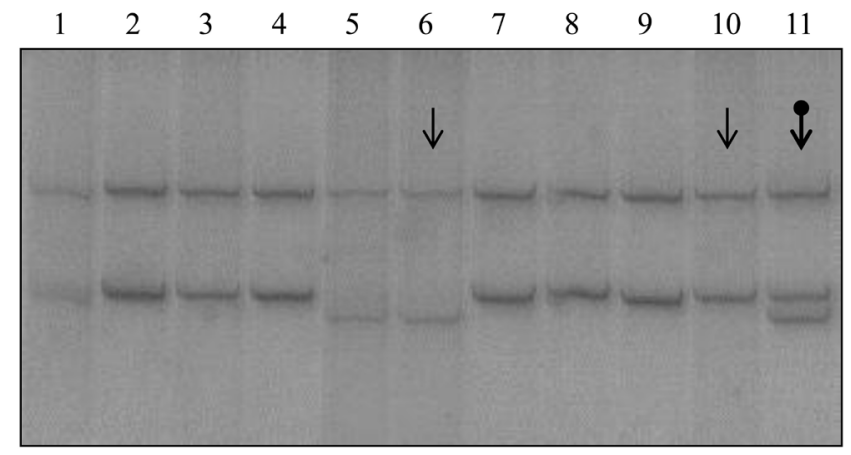

Figure 1. Screening of PRNP haplotype clones of each allele with SSCP. Two of different PRNP haplotype clones of each allele were screened with genomic PCR products of the SSCP banding patterns as a reference. Lanes 1 to $10=$ PCR-SSCP bands of PRNP haplotype clones; lane 11 = PCR-SSCP band of the PRNP allele. Lanes 6 and 10 $=$ two different clones of one PRNP allele were sequenced.

\section{ARMS-PCR analysis}

Three hundred and twenty-five sheep with unknown PRNP genotypes were detected by ARMS-PCR (Figure 2). PRNP genotypes determined by ARMS-PCR are shown in Table 3. The major genotypes of ARMS-PCR analysis were ARQ/ARQ, ARR/ARQ, ARR/ARR, ARQ/ ARH, ARK/ARQ, TRQ/ARQ, ARQ/AHQ, ARK/ARR, ARR/AHQ, ARR/VRR.

\section{Polymorphism of PRNP codons 136, 154 and 171}

Nineteen PRNP genotypes were detected by PCR-SSCP and ARMS-PCR in 757 sheep. The genotypes and the frequencies were as follows: ARQ/ARQ, 42.80\% (323/757); ARR/ARQ, 31.97\% (242/757); ARR/ARR, 15.32\% (116/757); ARQ/ARH, 2.77\% (21/757); ARH/ARH, 1.72\% (13/757); ARK/ARK, 1.06\% (8/757); ARK/ARQ, 0.92\% (7/757); VRQ/ ARQ, 0.66\% (5/757); TRQ/ARQ, 0.53\% (4/757); ARQ/AHQ, 0.53\% (4/757); ARK/ARR, 0.4\% (3/757); ARK/ARH, 0.26\% (2/757); TRQ/ARR, 0.26\% (2/757); AHR/ARQ, 0.13\% (1/757); ARR/ARH, 0.13\% (1/757); TRQ/TRQ, 0.13\% (1/757); AHQ/AHQ, 0.13\% (1/757); ARR/AHQ, 0.13\% (1/757), and ARR/VRR, 0.13\% (1/757) (Table 3). 
In all 17 detected genotypes, 90.09\% were ARQ/ARQ, ARR/ARQ and ARR/ARR, while 16 other genotypes only accounted for $9.91 \%$ (Table 3 ). Moreover, ARQ/ARQ itself accounted for $42.80 \%$, indicating that ARQ/ARQ may be the major genotype for sheep in this area. Indeed, ARQ/ARQ accounted for 83.36 and $70.27 \%$ in Qiaoke sheep and Oula sheep, respectively. It is noteworthy that Gansu modern meat sheep had the lowest percentage ARQ/ARQ; the major genotype in Gansu modern meat sheep was ARR/ARQ (83.68\%) (Table 3). The reason that the ARR/ARQ was strongly out of Hardy-Weinberg equilibrium remains to be clarified.

Table 3. Frequency of PRNP genotypes at codons 136, 154 and 171.

\begin{tabular}{|c|c|c|c|c|c|c|c|c|c|c|c|c|c|c|c|c|c|c|}
\hline \multirow[t]{2}{*}{ Genotypes } & \multicolumn{2}{|c|}{ STH sheep } & \multicolumn{2}{|c|}{ QK sheep } & \multicolumn{2}{|c|}{ Mon sheep } & \multicolumn{2}{|c|}{ Tan sheep } & \multicolumn{4}{|c|}{ GSAM } & \multicolumn{2}{|c|}{ OL sheep } & \multicolumn{2}{|c|}{ GMMP } & \multicolumn{2}{|c|}{ Total } \\
\hline & $\mathrm{N}$ & $\%$ & $\mathrm{~N}$ & $\%$ & $\mathrm{~N}$ & $\%$ & $\mathrm{~N}$ & $\%$ & $\mathrm{~N}$ & $\%$ & $\mathrm{~N}^{*}$ & $\% *$ & $N^{*}$ & $\% *$ & $\mathrm{~N}^{*}$ & $\% *$ & $\mathrm{~N}$ & $\%$ \\
\hline RQ/ARQ & 39 & 37.50 & 76 & 86.36 & 47 & 47.47 & 54 & 54.00 & 21 & 51.22 & 54 & 55.10 & 26 & 70.27 & 6 & 3.16 & 324 & 42.8 \\
\hline RR/ARQ & 12 & 11.54 & 3 & 3.41 & 10 & 10.10 & 13 & 13.00 & 13 & 31.71 & 29 & 29.59 & 3 & & 159 & 83.68 & 242 & 31.97 \\
\hline RR/ARR & 23 & 22.12 & 6 & 6.82 & 26 & 26.26 & 21 & 21.00 & 4 & 9.76 & 11 & 11.22 & 1 & & 24 & 12.63 & 116 & 15.32 \\
\hline $\mathrm{RQ} / \mathrm{ARH}$ & 11 & 10.58 & & 0.00 & 5 & 5.05 & 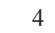 & 4.00 & 0 & 0.00 & 1 & & 0 & & 0 & & 21 & 2.77 \\
\hline RH/ARH & 0 & 1.92 & 3 & 3.4 & 5 & 5. & - & 2.00 & ? & 4.88 & 0 & & 0 & & 0 & & 13 & 1.72 \\
\hline RK/ARK & 1 & 0.9 & 0 & 0.0 & 4 & 4 & & 3. & 0 & 0.00 & 0 & 0 & 0 & 0 & 0 & 0 & 8 & 1.06 \\
\hline /ARQ & 4 & 3.85 & 0 & 0.0 & 0 & 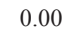 & 2 & 2. & & 0.00 & 0 & 0 & 1 & 2 & 0 & 0. & 7 & 0.92 \\
\hline $\mathrm{Q} / \mathrm{ARQ}$ & 5 & 4.81 & 0 & 0.0 & 0 & 0 & 0 & 0. & & 0.00 & 0 & & 0 & & 0 & 0. & 5 & 0.66 \\
\hline $\mathrm{Q} / \mathrm{ARQ}$ & 1 & 0.96 & 0 & 0.0 & 0 & 0 & 0 & 0. & $\sigma$ & 0.00 & $\sigma$ & 0 . & 3 & 8. & 0 & 0 . & 4 & 0.53 \\
\hline $\mathrm{RQ} / \mathrm{AHQ}$ & 0 & 0.00 & 0 & 0.00 & 0 & 00 & 0 & 0.00 & 1 & 2.44 & 2 & 2.04 & 1 & 2.70 & 0 & 0. & 4 & 0.53 \\
\hline ARK/ARR & 0 & 0.00 & 0 & 0.00 & 1 & 1.0 & 0 & 0.00 & 0 & 0.00 & 0 & 0.00 & 2 & 5.41 & 0 & 0.00 & 3 & 0.4 \\
\hline ARK/ARH & 2 & 1.92 & 0 & 0.00 & 0 & 0.0 & 0 & 0.00 & 0 & 0.00 & 0 & 0.00 & 0 & 0.00 & 0 & 0.00 & 2 & 0.26 \\
\hline TRQ/ARR & 1 & 0.96 & 0 & 0.00 & 1 & 1.01 & 0 & 0.00 & 0 & 0.00 & 0 & 0.00 & 0 & 0.00 & 0 & 0.00 & 2 & 0.26 \\
\hline AHR/ARQ & 1 & 0.96 & 0 & 0.00 & 0 & 0.0 & 0 & 0.00 & 0 & 0.00 & 0 & 0.00 & 0 & 0.00 & 0 & 0.00 & 1 & 0.13 \\
\hline ARR/ARH & 1 & 0.96 & 0 & 0.00 & 0 & 0.0 & 0 & 0.00 & 0 & 0.00 & 0 & 0.00 & 0 & 0.00 & 0 & 0.00 & 1 & 0.13 \\
\hline TRQ/TRQ & 1 & 0.96 & 0 & 0.00 & 0 & 0.0 & 0 & 0.00 & 0 & 0.00 & 0 & 0.00 & 0 & 0.00 & 0 & 0.00 & 1 & 0.13 \\
\hline $\mathrm{AHQ} / \mathrm{AHQ}$ & 0 & 0.00 & 0 & 0.00 & 0 & & 1 & 1.00 & 0 & 0.00 & 0 & 0 . & 0 & & 0 & 0.00 & 1 & 0.13 \\
\hline ARR/AHQ & 0 & 0.00 & 0 & 0.0 & 0 & & 0 & 0.00 & 0 & 0.00 & 1 & & 0 & & 0 & 0.00 & 1 & 0.13 \\
\hline ARR/VRR & 0 & 0.00 & 0 & 0.00 & 0 & 0.00 & 0 & 0.00 & 0 & 0.00 & 0 & 0.00 & 0 & 0.00 & 1 & 0.53 & 1 & 0.13 \\
\hline Total & 104 & & 88 & & 99 & & 100 & & 41 & & 98 & & 37 & & 190 & & 757 & \\
\hline
\end{tabular}

STH sheep = Small Tail Han sheep; QK sheep = Qiaoke sheep; Mon sheep = Mongolian sheep; GSAM = Gansu Alpine Merino; OL sheep = Oula sheep; GMMP = Gansu modern meat sheep new population; DNA fragments of PRNP alleles from 432 sheep were amplified and two clones of the haplotypes for each allele were also obtained. Three hundred and twenty-five sheep with unknown PRNP genotypes were detected by ARMS-PCR. *Genotyped by ARMS method. The major genotypes of ARMS-PCR analysis were ARQ/ARQ, ARR/ARQ, ARR/ARR, ARQ/ ARH, ARK/ARQ, TRQ/ARQ, ARQ/AHQ, ARK/ARR, ARR/AHQ, ARR/VRR. In all 17 genotypes detected, 90.09\% were ARQ/ARQ, ARR/ARQ and ARR/ARR, while other 16 genotypes only accounted for $9.91 \%$.

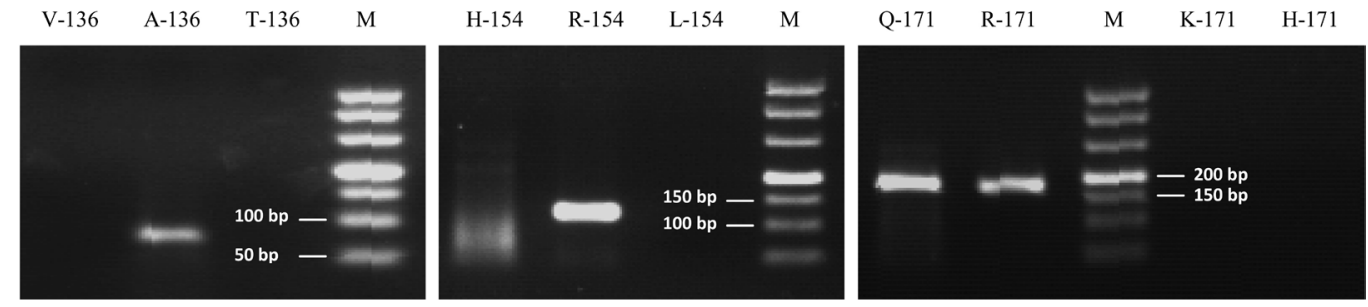

Figure 2. Genotyping of the sheep PRNP allele unknown genotype using the ARMS-PCR method. ARMS-PCR analysis was performed based on PCR-SSCP results, and was used to sheep PRNP genotyping unknow genotypes.

\section{Mutations in other codons}

A total of 24 mutations at 22 codons besides 136, 154 and 171 were detected with 
PCR-SSCP. The mutations were: G127V/S, G134R, M137T, S138N, L141F, H143R, N146S, E149V, H151C, M157V/T, Q163R, V164A，R167K/S，P168L，V169A，N174S, V183A, N184D, I185V/T, G189L, T195S, and E199V. Among them, G127V/S, M137T, S138N, L141F, H143R, N146S, H151C, R167S, P168L, G189L, and T195S were previously reported; however, G134R, E149V, M157V/T, Q163R, V164A, R167K/G, V169A, N174S, V183A, N184D, $\mathrm{I} 185 \mathrm{~V} / \mathrm{T}$, and $\mathrm{E} 199 \mathrm{~V}$ were found for the first time. Other reported mutations, such as G127A, Y152F, Q175E, N176K, H180Y, and G189R, were not found in these breeds.

The mutations in different genotypes of 136, 154 and 171 were quite different, all mutations outside 136, 154 and 171 and the genotypes are shown in Table 4.

\section{DISCUSSION}

In the present study, ARMS-PCR and PCR-SSCP were applied to determine the ovine PRNP allele or haplotype genotypes at codons 136, 154 and 171. These methods are reliable and more cost-effective compared to other approaches, including PCR-restriction fragment length polymorphism analysis (RFLP) (Hunter et al., 1991, 1993; Babar et al., 2009), denaturing gradient gel electrophoresis (DGGE) (Belt et al., 1995), direct sequencing (Tranulis et al., 1999; Baylis et al., 2002), high-throughput genotyping matrix assisted laser desorption/ ionization-time of flight (MALDI-TOF) systems (Humeny et al., 2002; Vollmert et al., 2006; Lee et al., 2007), allele-specific oligonucleotide hybridization (Hunter et al., 1997; Ishiguro et al., 1998), and primer extension assay (Zsolnai et al., 2003; Vaccari et al., 2004).

PCR-SSCP and ARMS-PCR have been used to detect genotypes (Buitkamp and Semmer, 2004; Zhou et al., 2005). In a previous study, amplified products of the unique SSCP patterns were cloned into a T-vector and were then sequenced (Zhou et al., 2005). However, in the present study, we detected so large numbers of other mutations in a preliminary experiment in which all PRNP alleles were sequenced. Genotyping results proved that our method is much more suitable in this study because of the superiority in analyzing different genotypes with similar banding patterns. We also improved the previous ARMS-PCR (Buitkamp et al., 2004) by developing a series of easier test procedures but not labeling primer with fluorochromes. Our improved ARMS-PCR detected mutations at codons 136, 154 and 171 such as $136 \mathrm{~T}, 154 \mathrm{~L}$ and $171 \mathrm{~K}$, which could not be detected by the previous method described by Buitkamp et al. (2004).

Based on PCR-SSCP results, we conducted ARMS-PCR analysis. The results showed that there were no mutations located in ARMS-PCR primer binding sequences and confirmed that the PRNP genotypes could not be detected by direct DNA sequencing method because of multiple nucleotide mutations of sheep PRNP alleles. Therefore, PCR-SSCP is the best method to detect mutations of sheep PRNP alleles, which is supported by the detection of large numbers of mutations outside condons 136, 154 and 171. Another advantage of PCR-SSCP is that the cost of SSCP is still very low compared to other methods, despite the costs in analyzing the relationship between the mutations, PRNP genotypes and sequences of different genotypes. However, PCR-SSCP was affected by many factors and the optimum conditions were not easily obtained. In addition, a long time was required for PCR-SSCP analysis, which reduced genotyping efficiency. However, for ARMS-PCR, as long as the method is established, cost of genotyping will be greatly reduced and genotyping efficiency will be greatly improved. Furthermore, ARMS-PCR was easy to perform, even for those with little experimental back- 
Table 4. Frequency of PRNP genotypes at codons 136, 154 and 171.

\begin{tabular}{|c|c|c|c|c|c|c|c|c|c|c|}
\hline \multirow[t]{2}{*}{ Genoytypes } & \multicolumn{2}{|c|}{$\begin{array}{l}\text { STH sheep } \\
(\mathrm{N}=104)\end{array}$} & \multicolumn{2}{|c|}{$\begin{array}{c}\text { QK sheep } \\
(\mathrm{N}=88)\end{array}$} & \multicolumn{2}{|c|}{$\begin{array}{l}\text { Mon sheep } \\
(\mathrm{N}=99)\end{array}$} & \multicolumn{2}{|c|}{$\begin{array}{l}\text { Tan sheep } \\
(\mathrm{N}=100)\end{array}$} & \multicolumn{2}{|c|}{$\begin{array}{l}\text { GSAM } \\
(\mathrm{N}=41)\end{array}$} \\
\hline & $\mathrm{N}$ & $\%$ & $\mathrm{~N}$ & $\%$ & $\mathrm{~N}$ & $\%$ & $\mathrm{~N}$ & $\% 0$ & $\mathrm{~N}$ & $\%$ \\
\hline $\mathrm{ARQ} / \mathrm{S}_{127} \mathrm{ARQ}$ & 0 & 0.00 & 4 & 45.45 & 2 & 20.20 & 4 & 40.00 & 0 & 0.00 \\
\hline $\mathrm{S}_{127} \mathrm{ARQ} / \mathrm{S}_{127}$ ARQ & 0 & 0.00 & 2 & 22.73 & 0 & 0.00 & 4 & 40.00 & 0 & 0.00 \\
\hline $\mathrm{ARR} / \mathrm{S}_{127} \mathrm{ARQ}$ & 0 & 0.00 & 1 & 11.36 & 0 & 0.00 & 2 & 20.00 & 0 & 0.00 \\
\hline $\mathrm{ARQL}_{189} / \mathrm{S}_{127} \mathrm{ARQ}$ & 0 & 0.00 & 1 & 11.36 & 0 & 0.00 & 1 & 10.00 & 0 & 0.00 \\
\hline $\mathrm{ARQ} / \mathrm{V}_{127} \mathrm{ARQ}$ & 0 & 0.00 & 0 & 0.00 & 0 & 0.00 & 1 & 10.00 & 0 & 0.00 \\
\hline $\mathrm{ARQ} / \mathrm{R}_{134} \mathrm{ARQ}$ & 2 & 19.23 & 1 & 11.36 & 2 & 20.20 & 0 & 0.00 & 0 & 0.00 \\
\hline $\mathrm{ARQL}_{189} / \mathrm{R}_{134} \mathrm{ARQ}$ & 0 & 0.00 & 1 & 11.36 & 1 & 10.10 & 0 & 0.00 & 0 & 0.00 \\
\hline $\mathrm{ARQ} / \mathrm{AT}_{137} \mathrm{RQ}$ & 0 & 0.00 & 0 & 0.00 & 0 & 0.00 & 3 & 30.00 & 0 & 0.00 \\
\hline ARQ/AN138RQ & 0 & 0.00 & 0 & 0.00 & 1 & 10.10 & 0 & 0.00 & 0 & 0.00 \\
\hline $\mathrm{ARQ} / \mathrm{AF}_{141} \mathrm{RQ}$ & 1 & 9.62 & 0 & 0.00 & 0 & 0.00 & 1 & 10.00 & 0 & 0.00 \\
\hline $\mathrm{AF}_{141} \mathrm{RQ} / \mathrm{ARQL}{ }_{189}$ & 2 & 19.23 & 0 & 0.00 & 0 & 0.00 & 0 & 0.00 & 0 & 0.00 \\
\hline $\mathrm{ARQ} / \mathrm{AR}_{143} \mathrm{RQ}$ & 0 & 0.00 & 0 & 0.00 & 0 & 0.00 & 1 & 10.00 & 3 & 73.17 \\
\hline $\mathrm{ARR} / \mathrm{AR}_{143}{ }^{143} \mathrm{RQ}$ & 0 & 0.00 & 0 & 0.00 & 1 & 10.10 & 1 & 10.00 & 2 & 48.78 \\
\hline $\mathrm{ARQ} / \mathrm{AS}_{146} \mathrm{RQ}$ & 0 & 0.00 & 1 & 11.36 & 0 & 0.00 & 0 & 0.00 & 0 & 0.00 \\
\hline $\mathrm{ARQL}_{189} / \mathrm{AS}_{146} \mathrm{RQ}$ & 0 & 0.00 & 0 & 0.00 & 1 & 10.10 & 0 & 0.00 & 1 & 24.39 \\
\hline $\mathrm{ARR} / \mathrm{AS}_{146} \mathrm{RQ}$ & 0 & 0.00 & 0 & 0.00 & 0 & 0.00 & 0 & 0.00 & 2 & 48.78 \\
\hline $\mathrm{AS}_{146} \mathrm{RR} / \mathrm{AS}_{146} \mathrm{RQ}$ & 0 & 0.00 & 0 & 0.00 & 0 & 0.00 & 0 & 0.00 & 1 & 24.39 \\
\hline $\mathrm{ARQ} / \mathrm{AC}_{151} \mathrm{RQ}^{140}$ & 0 & 0.00 & 0 & 0.00 & 0 & 0.00 & 0 & 0.00 & 1 & 24.39 \\
\hline $\mathrm{ARQ} / \mathrm{ART}_{157} \mathrm{Q}$ & 1 & 9.62 & 0 & 0.00 & 1 & 10.10 & 0 & 0.00 & 0 & 0.00 \\
\hline $\mathrm{ARQL}_{189} / \mathrm{ART}_{157} \mathrm{Q}$ & 0 & 0.00 & 0 & 0.00 & 0 & 0.00 & 0 & 0.00 & 1 & 24.39 \\
\hline $\mathrm{ARQ} / \mathrm{ARH}_{158} \mathrm{Q}$ & 0 & 0.00 & 0 & 0.00 & 0 & 0.00 & 0 & 0.00 & 1 & 24.39 \\
\hline $\mathrm{ARR}_{163} \mathrm{Q} / \mathrm{ARQL}_{189}$ & 0 & 0.00 & 1 & 11.36 & 1 & 10.10 & 0 & 0.00 & 0 & 0.00 \\
\hline $\mathrm{ARR}_{163}{ }^{163} \mathrm{Q} / \mathrm{ARQ}$ & 0 & 0.00 & 0 & 0.00 & 1 & 10.10 & 0 & 0.00 & 0 & 0.00 \\
\hline $\mathrm{ARQ} / \mathrm{ARA}_{164} \mathrm{Q}$ & 0 & 0.00 & 1 & 11.36 & 0 & 0.00 & 0 & 0.00 & 1 & 24.39 \\
\hline $\mathrm{ARQ} / \mathrm{ARK}_{167} \mathrm{Q}$ & 0 & 0.00 & 1 & 11.36 & 0 & 0.00 & 2 & 20.00 & 0 & 0.00 \\
\hline $\mathrm{ARQ} / \mathrm{ARS}_{167} \mathrm{Q}$ & 0 & 0.00 & 0 & 0.00 & 0 & 0.00 & 1 & 10.00 & 0 & 0.00 \\
\hline $\mathrm{ARQ} / \mathrm{ARL}_{168} \mathrm{Q}$ & 0 & 0.00 & 0 & 0.00 & 0 & 0.00 & 0 & 0.00 & 1 & 24.39 \\
\hline $\mathrm{ARQ} / \mathrm{ARA}_{169}{ }_{169} \mathrm{Q}$ & 0 & 0.00 & 2 & 22.73 & 0 & 0.00 & 0 & 0.00 & 1 & 24.39 \\
\hline $\mathrm{ARQL}_{189} / \mathrm{ARA}_{169} \mathrm{Q}$ & 0 & 0.00 & 1 & 11.36 & 0 & 0.00 & 0 & 0.00 & 0 & 0.00 \\
\hline $\mathrm{ARR} / \mathrm{ARA}_{169} \mathrm{Q}$ & 0 & 0.00 & 0 & 0.00 & 0 & 0.00 & 1 & 10.00 & 0 & 0.00 \\
\hline $\mathrm{ARQ} \mathrm{ARQS}_{174}$ & 0 & 0.00 & 0 & 0.00 & 1 & 10.10 & 0 & 0.00 & 0 & 0.00 \\
\hline $\mathrm{ARQL}_{189} / \mathrm{ARQD}_{184} \mathrm{~L}_{189}$ & 0 & 0.00 & 1 & 11.36 & 0 & 0.00 & 0 & 0.00 & 0 & 0.00 \\
\hline $\mathrm{ARQ} / \mathrm{ARQV} V_{185}$ & 0 & 0.00 & 0 & 0.00 & 0 & 0.00 & 1 & 10.00 & 2 & 48.78 \\
\hline $\mathrm{ARQ} \mathrm{ARQT}_{185}$ & 0 & 0.00 & 0 & 0.00 & 0 & 0.00 & 0 & 0.00 & 1 & 24.39 \\
\hline ARH/ARQL ${ }_{189}$ & 2 & 19.23 & 2 & 22.73 & 0 & 0.00 & 1 & 10.00 & 1 & 24.39 \\
\hline $\mathrm{ARQ} / \mathrm{AR}_{\mathrm{QL} 1} 89$ & 4 & 38.46 & 11 & 125.00 & 3 & 30.30 & 4 & 40.00 & 3 & 73.17 \\
\hline $\mathrm{ARK} / \mathrm{ARQL}_{189}$ & 0 & 0.00 & 1 & 11.36 & 0 & 0.00 & 1 & 10.00 & 2 & 48.78 \\
\hline $\mathrm{ARQL}_{189} / \mathrm{ARQL}_{189}$ & 6 & 57.69 & 16 & 181.82 & 5 & 50.51 & 7 & 70.00 & 4 & 97.56 \\
\hline $\mathrm{ARR} \mathrm{ARQL} \mathrm{L}_{189}$ & 0 & 0.00 & 5 & 56.82 & 0 & 0.00 & 2 & 20.00 & 0 & 0.00 \\
\hline TRQ/ARQL & 0 & 0.00 & 0 & 0.00 & 0 & 0.00 & 0 & 0.00 & 1 & 24.39 \\
\hline $\mathrm{ARQ} \mathrm{ARQS}_{195}$ & 0 & 0.00 & 0 & 0.00 & 1 & 10.10 & 1 & 10.00 & 0 & 0.00 \\
\hline $\mathrm{ARQ} / \mathrm{ARQV}_{199}$ & 2 & 19.23 & 0 & 0.00 & 0 & 0.00 & 0 & 0.00 & 0 & 0.00 \\
\hline $\mathrm{ARR} / \mathrm{S}_{127} \mathrm{ARR}$ & 0 & 0.00 & 0 & 0.00 & 2 & 20.20 & 0 & 0.00 & 0 & 0.00 \\
\hline $\mathrm{ARQ} / \mathrm{AT}_{137} \mathrm{RR}$ & 0 & 0.00 & 0 & 0.00 & 0 & 0.00 & 1 & 10.00 & 0 & 0.00 \\
\hline $\mathrm{ARQ} / \mathrm{AF}_{141}^{13 /} \mathrm{RR}$ & 0 & 0.00 & 0 & 0.00 & 2 & 20.20 & 0 & 0.00 & 0 & 0.00 \\
\hline $\mathrm{ARQ} / \mathrm{AS}_{146} \mathrm{RR}$ & 0 & 0.00 & 0 & 0.00 & 0 & 0.00 & 0 & 0.00 & 1 & 24.39 \\
\hline $\mathrm{ARR} / \mathrm{AC}_{151}{ }^{140} \mathrm{RR}$ & 0 & 0.00 & 0 & 0.00 & 0 & 0.00 & 0 & 0.00 & 1 & 24.39 \\
\hline $\mathrm{ARQ} / \mathrm{AC}_{151} \mathrm{RR}$ & 0 & 0.00 & 0 & 0.00 & 0 & 0.00 & 0 & 0.00 & 1 & 24.39 \\
\hline $\mathrm{ARQ} / \mathrm{ARV}_{157}{ }_{15} \mathrm{R}$ & 0 & 0.00 & 0 & 0.00 & 0 & 0.00 & 1 & 10.00 & 0 & 0.00 \\
\hline $\mathrm{ARQ} / \mathrm{ARA}_{164} \mathrm{R}$ & 0 & 0.00 & 0 & 0.00 & 1 & 10.10 & 0 & 0.00 & 0 & 0.00 \\
\hline $\mathrm{ARR} \mathrm{ARRS}_{174}$ & 0 & 0.00 & 0 & 0.00 & 0 & 0.00 & 1 & 10.00 & 0 & 0.00 \\
\hline $\mathrm{AHQ} / \mathrm{ARRA}_{183}$ & 0 & 0.00 & 0 & 0.00 & 2 & 20.20 & 0 & 0.00 & 1 & 24.39 \\
\hline $\mathrm{ARQ} \mathrm{ARRT}_{185}$ & 0 & 0.00 & 0 & 0.00 & 0 & 0.00 & 0 & 0.00 & 2 & 48.78 \\
\hline ARQ/ARRL & 1 & 9.62 & 1 & 11.36 & 1 & 10.10 & 1 & 10.00 & 1 & 24.39 \\
\hline $\mathrm{ARR}$ ARRV ${ }_{199}$ & 0 & 0.00 & 0 & 0.00 & 1 & 10.10 & 0 & 0.00 & 0 & 0.00 \\
\hline $\mathrm{ARQ} / \mathrm{AR}_{143} \mathrm{RH}$ & 0 & 0.00 & 0 & 0.00 & 0 & 0.00 & 0 & 0.00 & 1 & 24.39 \\
\hline $\mathrm{ARH} \mathrm{ARV}_{157} \mathrm{H}$ & 0 & 0.00 & 1 & 11.36 & 0 & 0.00 & 0 & 0.00 & 0 & 0.00 \\
\hline $\mathrm{ARQ} / \mathrm{ARG}_{167}^{157} \mathrm{H}$ & 1 & 9.62 & 0 & 0.00 & 0 & 0.00 & 0 & 0.00 & 1 & 24.39 \\
\hline $\mathrm{ARG}_{167} \mathrm{H} / \mathrm{ARQL}_{189}$ & 1 & 9.62 & 0 & 0.00 & 0 & 0.00 & 0 & 0.00 & 0 & 0.00 \\
\hline
\end{tabular}

Continued on next page 


\begin{tabular}{|c|c|c|c|c|c|c|c|c|c|c|}
\hline \multirow[t]{2}{*}{ Genoytypes } & \multicolumn{2}{|c|}{$\begin{array}{l}\text { STH sheep } \\
(\mathrm{N}=104)\end{array}$} & \multicolumn{2}{|c|}{$\begin{array}{c}\text { QK sheep } \\
(\mathrm{N}=88)\end{array}$} & \multicolumn{2}{|c|}{$\begin{array}{l}\text { Mon sheep } \\
\quad(\mathrm{N}=99)\end{array}$} & \multicolumn{2}{|c|}{$\begin{array}{l}\text { Tan sheep } \\
(\mathrm{N}=100)\end{array}$} & \multicolumn{2}{|c|}{$\begin{array}{l}\text { GSAM } \\
(\mathrm{N}=41)\end{array}$} \\
\hline & $\mathrm{N}$ & $\%$ & $\mathrm{~N}$ & $\%$ & $\mathrm{~N}$ & $\%$ & $\mathrm{~N}$ & $\%$ & $\mathrm{~N}$ & $\%$ \\
\hline $\mathrm{ARQL}_{189} / \mathrm{ARHA}_{183}$ & 0 & 0.00 & 0 & 0.00 & 1 & 10.10 & 0 & 0.00 & 0 & 0.00 \\
\hline $\mathrm{ARQ} / \mathrm{ARHA}{ }_{183}$ & 0 & 0.00 & 0 & 0.00 & 1 & 10.10 & 0 & 0.00 & 0 & 0.00 \\
\hline $\mathrm{ARQ} \mathrm{ARHL}_{189}$ & 2 & 19.23 & 2 & 22.73 & 0 & 0.00 & 0 & 0.00 & 1 & 24.39 \\
\hline $\mathrm{ARQ} / \mathrm{AT}_{137} \mathrm{RK}$ & 0 & 0.00 & 0 & 0.00 & 1 & 10.10 & 0 & 0.00 & 0 & 0.00 \\
\hline $\mathrm{ARQ} / \mathrm{AV}_{149}{ }^{13} \mathrm{RK}$ & 0 & 0.00 & 0 & 0.00 & 1 & 10.10 & 0 & 0.00 & 0 & 0.00 \\
\hline $\mathrm{ARK} / \mathrm{ARG}_{167} \mathrm{~K}$ & 0 & 0.00 & 0 & 0.00 & 1 & 10.10 & 0 & 0.00 & 0 & 0.00 \\
\hline ARR/ARL ${ }_{168}^{167} \mathrm{~K}$ & 0 & 0.00 & 0 & 0.00 & 1 & 10.10 & 0 & 0.00 & 0 & 0.00 \\
\hline $\mathrm{ARK} / \mathrm{TRQL}_{189}$ & 1 & 9.62 & 0 & 0.00 & 0 & 0.00 & 0 & 0.00 & 0 & 0.00 \\
\hline $\mathrm{AHQ} / \mathrm{AHQL}_{189}$ & 0 & 0.00 & 0 & 0.00 & 0 & 0.00 & 0 & 0.00 & 1 & 24.39 \\
\hline
\end{tabular}

STH sheep = Small Tail Han sheep; QK sheep = Qiaoke sheep; Mon sheep = Mongolian sheep; GSAM = Gansu Alpine Merino. All the frequencies of PRNP alleles were shown in permillage. A total of 24 mutations at 22 codons besides 136, 154 and 171 were detected with PCR-SSCP. Among mutations, G127V/S, M137T, S138N, L141F, H143R, N146S, H151C, R167S, P168L, G189L, and T195S were reported previously; G134R, E149V, M157V/T, Q163R, V164A, R167K/G, V169A, N174S, V183A, N184D, I185V/T, and E199V were found for the first time. Other reported mutations, such as G127A, Y152F, Q175E, N176K, H180Y, and G189R were not found in these breeds.

ground. It is worth noting that large numbers of known haplotypes of alleles were required for establishing ARMS-PCR, and this problem could be solved by the separation of haplotypes by PCR-SSCP and haplotype sequencing. If haplotypes were not separated by SSCP, genotypes with more than two mutations could not be genotyped and only the kinds and location of the mutations could be detected. We were eager to develop an inexpensive and efficient method for fast PRNP genotyping, so ARMS-PCR became the preferred method. For these reasons, the three typing methods PCR-SSCP, ARMS-PCR and direct sequencing were involved.

Nineteen ovine PRNP genotypes and 24 mutations at 22 codons besides 136, 154 and 171 were detected in 757 sheep from seven different breeds. The VRQ/VRQ genotype was absent and ARQ/VRQ shared a low frequency in this study, indicating that China will be safe from the most virulent of the sheep scrapie strains (Langeveld et al., 2006; Marcos-Carcavilla et al., 2010). It is worth noting that the genotypes such as AHQ/AHQ, ARQ/AHQ, ARH/ ARH, ARQ/ARH, ARQ/ARQ, and VRQ/ARQ, accounting for 48.61\% (368/757), are highly susceptible to scrapie, and the scrapie-resistant genotype ARR/ARR only accounts for $15.32 \%$ (116/757). Therefore, sheep populations in northwestern China have a high risk of scrapie infection (Lezmi et al., 2006; Tongue et al., 2006), especially when scrapie occurs in neighboring countries. This is supported by other studies that showed a high percentage of ARQ/ ARQ in China (Zhang et al., 2004; Lan et al., 2006). Therefore, a sheep anti-scrapie breeding program is imperative, not only for animal health of sheep, cattle and other ruminants, but also for public health safety.

The percentage of ARQ/ARQ in Qiaoke sheep and Oula sheep is higher than that in the other groups, indicating that there is a high risk of scrapie infection in Qiaoke sheep and Oula sheep. We also found the lowest percentage of ARQ/ARQ and the highest percentage of ARR/ ARQ (up to 83.68\%) in Gansu modern meat sheep new population. In the breeding process, lots of ram were eliminated according to the breeding targets such as roughage-resistance, little size and high growth rate, which would lead to the retained rams having a higher ARR/ARR and ewes having a higher ARQ/ARQ. After selection breeding, the frequency of ARQ/ARR in descendants was higher than ARQ/ARQ and ARR/ARR, which was out of Hardy-Weinberg equilibrium. 


\section{ACKNOWLEDGMENTS}

Research supported by the Specialized Research Fund for the Doctoral Program of Higher Education (SRFDP, \#20060733006), the State Key Laboratory of Veterinary Etiological Biology, Lanzhou Veterinary Research Institute, Chinese Academy of Agricultural Sciences (\#2008-2009), the Key Projects in the National Science \& Technology Pillar Program during the Eleventh Five-Year Plan Period (\#2008BAD2B04), and the Earmarked Fund for Modern Agro-Industry Technology Research System (\#2008-2012).

\section{REFERENCES}

Andreoletti O, Morel N, Lacroux C, Rouillon V, et al. (2006). Bovine spongiform encephalopathy agent in spleen from an ARR/ARR orally exposed sheep. J. Gen. Virol. 87: 1043-1046.

Babar ME, Abdullah M, Nadeem A and Haq AU (2009). Prion protein gene polymorphisms in four goat breeds of Pakistan. Mol. Biol. Rep. 36: 141-144.

Baylis M, Goldmann W, Houston F, Cairns D, et al. (2002). Scrapie epidemic in a fully PrP-genotyped sheep flock. $J$. Gen. Virol. 83: 2907-2914.

Belt PB, Muileman IH, Schreuder BE, Bos-de Ruijter J, et al. (1995). Identification of five allelic variants of the sheep PrP gene and their association with natural scrapie. J. Gen. Virol. 76: 509-517.

Buitkamp J and Semmer J (2004). A robust, low- to medium-throughput prnp genotyping system in sheep. BMC Infect. Dis. 4: 30.

De Vries F, Borchers N, Hamann H, Drogemuller C, et al. (2004). Associations between the prion protein genotype and performance traits of meat breeds of sheep. Vet. Rec. 155: 140-143.

Goldmann W, Houston F, Stewart P, Perucchini M, et al. (2006). Ovine prion protein variant $\mathrm{A}^{136} \mathrm{R}^{154} \mathrm{~L}^{168} \mathrm{Q}^{171}$ increases resistance to experimental challenge with bovine spongiform encephalopathy agent. J. Gen. Virol. 87: 3741-3745.

Hagenaars TJ, Donnelly CA and Ferguson NM (2006). Epidemiological analysis of data for scrapie in Great Britain. Epidemiol. Infect. 134: 359-367.

Humeny A, Schiebel K, Seeber S and Becker CM (2002). Identification of polymorphisms within the bovine prion protein gene (Prnp) by DNA sequencing and genotyping by MALDI-TOF-MS. Neurogenetics 4: 59-60.

Hunter N (1997). Molecular Biology and Genetics of Scrapie in Sheep. In: The Genetics of Sheep. (Piper L and Ruvinsky A, eds.). CAB International, Wallingford, 225-240.

Hunter N, Foster JD, Benson G and Hope J (1991). Restriction fragment length polymorphisms of the scrapie-associated fibril protein $(\mathrm{PrP})$ gene and their association with susceptibility to natural scrapie in British sheep. J. Gen. Virol. 72: $1287-1292$.

Hunter N, Goldmann W, Benson G, Foster JD, et al. (1993). Swaledale sheep affected by natural scrapie differ significantly in PrP genotype frequencies from healthy sheep and those selected for reduced incidence of scrapie. J. Gen. Virol. 74: 1025-1031.

Hunter N, Moore L, Hosie BD, Dingwall WS, et al. (1997). Association between natural scrapie and PrP genotype in a flock of Suffolk sheep in Scotland. Vet. Rec. 140: 59-63.

Ishiguro N, Shinagawa M, Onoe S, Yamanouchi K, et al. (1998). Rapid analysis of allelic variants of the sheep PrP gene by oligonucleotide probes. Microbiol. Immunol. 42: 579-582.

Lan Z, Wang ZL, Liu Y and Zhang X (2006). Prion protein gene (PRNP) polymorphisms in Xinjiang local sheep breeds in China. Arch. Virol. 151: 2095-2101.

Langeveld JP, Jacobs JG, Erkens JH, Bossers A, et al. (2006). Rapid and discriminatory diagnosis of scrapie and BSE in retro-pharyngeal lymph nodes of sheep. BMC Vet. Res. 2: 19.

Lee MA, Manley TR, Glass BC, Anderson RM, et al. (2007). Distribution of prion protein genotypes in breeds of sheep in New Zealand. N. Z. Vet. J. 55: 222-227.

Lezmi S, Ronzon F, Bencsik A, Bedin A, et al. (2006). PrP(d) accumulation in organs of ARQ/ARQ sheep experimentally infected with BSE by peripheral routes. Acta Biochim. Pol. 53: 399-405.

Li YM and Tian B (2002). Chinese little-fat-tail sheep prion protein gene belongs to PrPARH genotype. Sheng Wu Hua Xue. Yu Sheng Wu Wu Li Xue Bao 34: 62-66.

Lipsky S, Brandt H, Luhken G and Erhardt G (2008). Analysis of prion protein genotypes in relation to reproduction traits in local and cosmopolitan German sheep breeds. Anim. Reprod. Sci. 103: 69-77. 
Marcos-Carcavilla A, Moreno C, Serrano M, Laurent P, et al. (2010). Polymorphisms in the HSP90AA1 5' flanking region are associated with scrapie incubation period in sheep. Cell Stress Chaperones 15: 343-349.

Melchior MB, Windig JJ, Hagenaars TJ, Bossers A, et al. (2010). Eradication of scrapie with selective breeding: are we nearly there? BMC Vet. Res. 6: 24.

Sanguinetti CJ, Dias NE and Simpson AJ (1994). Rapid silver staining and recovery of PCR products separated on polyacrylamide gels. Biotechniques 17: 914-921.

Sawalha RM, Brotherstone S, Man WY, Conington J, et al. (2007). Associations of polymorphisms of the ovine prion protein gene with growth, carcass, and computerized tomography traits in Scottish Blackface lambs. J. Anim. Sci. 85: 632-640.

Sweeney T, Hanrahan JP and O'Doherty E (2007). Is there a relationship between prion protein genotype and ovulation rate and litter size in sheep? Anim. Reprod. Sci. 101: 153-157.

Tongue SC, Pfeiffer DU, Warner R, Elliott H, et al. (2006). Estimation of the relative risk of developing clinical scrapie: the role of prion protein (PrP) genotype and selection bias. Vet. Rec. 158: 43-50.

Tranulis MA, Osland A, Bratberg B and Ulvund MJ (1999). Prion protein gene polymorphisms in sheep with natural scrapie and healthy controls in Norway. J. Gen. Virol. 80: 1073-1077.

Vaccari G, Conte M, Morelli L, Di Guardo G, et al. (2004). Primer extension assay for prion protein genotype determination in sheep. Mol. Cell Probes 18: 33-37.

Vitezica ZG, Moreno CR, Lantier F, Lantier I, et al. (2007). Quantitative trait loci linked to PRNP gene controlling health and production traits in INRA 401 sheep. Genet. Sel. Evol. 39: 421-430.

Vollmert C, Windl O, Xiang W, Rosenberger A, et al. (2006). Significant association of a M129V independent polymorphism in the 5' UTR of the PRNP gene with sporadic Creutzfeldt-Jakob disease in a large German casecontrol study. J. Med. Genet. 43: e53.

Zhang L, Li N, Fan B, Fang M, et al. (2004). PRNP polymorphisms in Chinese ovine, caprine and bovine breeds. Anim. Genet. 35: 457-461.

Zhou H, Hickford JG and Fang Q (2005). Technical note: determination of alleles of the ovine PRNP gene using PCRsingle-strand conformational polymorphism analysis. J. Anim. Sci. 83: 745-749.

Zsolnai A, Anton I, Kuhn C and Fesus L (2003). Detection of single-nucleotide polymorphisms coding for three ovine prion protein variants by primer extension assay and capillary electrophoresis. Electrophoresis 24: 634-638. 